\title{
Apoplastic Nucleoside Accumulation in Arabidopsis Leads to Reduced Photosynthetic Performance and Increased Susceptibility Against Botrytis cinerea
}

\author{
Manuel Daumann, Marietta Fischer, Sandra Niopek-Witz, Christopher Girke and \\ Torsten Möhlmann *
}

Pflanzenphysiologie, Fachbereich Biologie, Technische Universität Kaiserslautern, Kaiserslautern, Germany

\section{OPEN ACCESS}

Edited by:

Vincenzo Lionetti,

Sapienza-Università di Roma, Italy

Reviewed by:

Claus-Peter Witte,

Free University Berlin, Germany

Kiwamu Tanaka,

Washington State University, USA

${ }^{*}$ Correspondence:

Torsten Möhlmann

moehlmann@biologie.uni-kl.de

Specialty section: This article was submitted to

Plant Biotic Interactions,

a section of the journal

Frontiers in Plant Science

Received: 23 October 2015 Accepted: 07 December 2015 Published: 23 December 2015

Citation:

Daumann M, Fischer M,

Niopek-Witz S, Girke C and Möhlmann T (2015) Apoplastic

Nucleoside Accumulation in Arabidopsis Leads to Reduced Photosynthetic Performance and Increased Susceptibility Against Botrytis cinerea.

Front. Plant Sci. 6:1158. doi: 10.3389/fpls.2015.01158
Interactions between plant and pathogen often occur in the extracellular space and especially nucleotides like ATP and NAD have been identified as key players in this scenario. Arabidopsis mutants accumulating nucleosides in the extracellular space were generated and studied with respect to susceptibility against Botrytis cinerea infection and general plant fitness determined as photosynthetic performance. The mutants used are deficient in the main nucleoside uptake system ENT3 and the extracellular nucleoside hydrolase NSH3. When grown on soil but not in hydroponic culture, these plants markedly accumulate adenosine and uridine in leaves. This nucleoside accumulation was accompanied by reduced photosystem II efficiency and altered expression of photosynthesis related genes. Moreover, a higher susceptibility toward Botrytis cinerea infection and a reduced induction of pathogen related genes PR1 and WRKY33 was observed. All these effects did not occur in hydroponically grown plants substantiating a contribution of extracellular nucleosides to these effects. Whether reduced general plant fitness, altered pathogen response capability or more direct interactions with the pathogen are responsible for these observations is discussed.

Keywords: ENT3, NSH3, uridine, adenosine, photosynthesis, Botrytis cinerea, Arabidopsis thaliana

\section{INTRODUCTION}

Nucleotide metabolism is an essential process in all living organisms as nucleotides function as energy providers, building blocks for nucleic acids, as a signaling component and as precursor for the biosynthesis of the phytohormone cytokinin (Buchanan et al., 2002). Nucleotide metabolism can be roughly divided into three parts: (i) de novo synthesis, (ii) salvage of nucleosides and nucleobases, and (iii) catabolism of purines and pyrimidines (Zrenner et al., 2006). Latter requires different subsets of enzymes, for example nucleoside hydrolases (NSH), which initially degrade nucleosides to nucleobases by removing the ribose moiety. Cytosolic NSH1 was attributed a key regulator at the branch point between salvage processes fueling the nucleotide pool and pyrimidine catabolism (Jung et al., 2009). One member of the Arabidopsis NSH protein family, NSH3, belongs to the purine specific IAG-NH family (inosine-adenosine-guanosine nucleoside hydrolases) and was shown to accept adenosine and inosine as substrates (Jung et al., 2011). The results of two 
independent proteome analyses (Borderies et al., 2003; Kwon et al., 2005) as well as the analysis of apoplastic sap, identified NSH3 as an extracellular protein, assumed to function in extracellular nucleoside turnover (Jung et al., 2011). Incubation of seedlings with jasmonic acid leads to an increased $\mathrm{NSH} 3$ expression, indicating potential participation of NSH3 in plant wound or pathogen response (Taki et al., 2005; Jung et al., 2011).

In addition to catabolic enzymes, specific transport processes are necessary for proper nucleotide metabolism. Whereas several protein families are known to facilitate nucleobase transport (PUPs, NATs, AZGs), the equilibrative nucleoside transporter family (ENT, eight members in Arabidopsis thaliana) represents the only group of nucleoside transporters in plants so far (reviewed by Girke et al., 2014). Following a knockout screen of ENT proteins for resistance against toxic 5-fluoro-uridine, the plasma membrane located ENT3 transporter was identified as major nucleoside importer in Arabidopsis (Traub et al., 2007).

So far the origin of extracellular nucleosides is uncertain. They might be liberated by intact or wounded cells, emerge as degradation products of the extracellular messenger eATP (Möhlmann et al., 2014) or can be imported from the rhizosphere (Traub et al., 2007). As both, NSH3 and ENT3 are supposed to participate in shaping the extracellular nucleoside pool, the simultaneous knockout of both genes could be used for the comprehensive investigation of the origin, the role and the physiological functions of extracellular nucleosides.

Extracellular nucleotides (eATP, NAD) function as signals or damage-associated molecular patterns, DAMPS, which i.e., play a role in the plant pathogen response (Zhang and Mou, 2009; Tanaka et al., 2014). To analyze whether nucleosides can exert similar functions, pathogenicity tests are a suitable approach. Botrytis cinerea (B. cinerea) is a necrotrophic fungus with a broad host range and one of the best studied fungal organisms (Hahn, 2014).

The aim of this work was to provoke an accumulation of apoplastic nucleosides and to study the resulting effects. For this, double knockout plants of the major Arabidopsis nucleoside transporter ENT3 and the extracellular nucleoside hydrolase NSH3 were generated and analyzed. Furthermore, the source of apoplastic nucleosides was investigated by comparing leaf exudates from soil grown with hydroponically grown plants. As extracellular nucleotides and nucleosides are reported to affect pathogen responses of Arabidopsis, a pathogenicity test of the mutants was performed in addition to expression analysis of pathogenicity related genes and measurements of the plants photosynthetic performance.

\section{MATERIALS AND METHODS}

\section{Plant Growth}

Wild-type and transgenic Arabidopsis thaliana (L.) Heynh. plants (ecotype Columbia) were used throughout. Plants were grown in standardized ED73 (Einheitserde und Humuswerke Patzer, Buchenberg, Germany) soil under short day conditions $\left(120 \mu \mathrm{mol}\right.$ quanta $\mathrm{m}^{-2} \mathrm{~s}^{-1}$ in a $10 \mathrm{~h}$ light/14h dark regime, temperature $22^{\circ} \mathrm{C}$, humidity $60 \%$ ). Prior to germination, seeds were incubated for $24 \mathrm{~h}$ in the dark at $4^{\circ} \mathrm{C}$ for imbibition (Weigel and Glazebrook, 2002). Growth in Erlenmeyer glass flasks with 100 surface sterilized seed (standard liquid culture) was performed as described by Scheible et al. (2004). Composition of the medium as well as the nitrogen free medium was described earlier Jung et al. (2009). Latter was supplemented with $1 \mathrm{mM}$ inosine. For growth experiments with toxic nucleoside analogs, surface sterilized seeds were sown on half-strength Murashige and Skoog medium (MS, Sigma, Steinheim, Germany) containing 5-fluoro-uridine (Sigma, Steinheim, Germany) and 2-chloro-adenosine (Sigma, Steinheim, Germany) as described earlier (Jung et al., 2011). Growth of plants in hydroponic culture was performed following the description of Conn et al. (2013). Briefly, seeds were placed on microcentrifuge-tube lids containing germination medium $\left(0.75 \mathrm{mM} \mathrm{CaCl}_{2}, 1 \mathrm{mM} \mathrm{KCl}\right.$, $0.25 \mathrm{mM} \mathrm{Ca}\left(\mathrm{NO}_{3}\right)_{2}, 1 \mathrm{mM} \mathrm{MgSO}_{4}, 0.2 \mathrm{mM} \mathrm{KH}_{2} \mathrm{PO}_{4}, 0.7 \%$ agar, $\mathrm{pH}$ 5.6) arranged in a $250 \mathrm{ml}$ growth chamber. The insides of the lids were surrounded by liquid germination medium. After stratification as described above, plants were grown under short day conditions (see above) for 7 days. At day 8 the bath solution was adapted to standard nutrient solution over 3 days $(0.1 \mathrm{mM}$ $\mathrm{CaCl}_{2}, 2 \mathrm{mM} \mathrm{KCl}, 2 \mathrm{mM} \mathrm{Ca}\left(\mathrm{NO}_{3}\right)_{2}, 2 \mathrm{mM} \mathrm{MgSO}_{4}, 0.6 \mathrm{mM}$ $\left.\mathrm{KH}_{2} \mathrm{PO}_{4}, 1.5 \mathrm{mM} \mathrm{NaCl}, \mathrm{pH} 5.6\right)$. At day 21 the plants were transferred to $50 \mathrm{ml}$ centrifuge tubes and placed into $12 \mathrm{~L}$ tanks containing standard nutrient solution. All media additionally contained the following micronutrients: $50 \mu \mathrm{M} \mathrm{NaFe(III)EDTA,}$

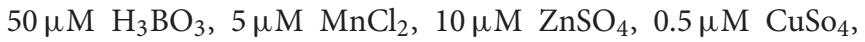
$0.1 \mu \mathrm{M} \mathrm{Na} \mathrm{MoO}_{3}$. Physiological analyses were performed with either 3, 4, or 6 week old plants. In addition to wild-type plants, ent3-1 (At4g05120; SALK\#131585) nsh3-1 (At5g18860; SAIL\#444_C09), and corresponding ent3:nsh3 double mutants were used (Traub et al., 2007; Jung et al., 2011).

\section{Mutant Screening}

Homozygous ent3:nsh3 mutants were identified in crossed populations of ent 3 and $n s h 3$ single mutants via PCR using genomic DNA templates. These mutants are characterized by the absence of a corresponding ENT3 or NSH3 gene specific PCR product which is obtained in wild-type plants with the primer combinations ENT3 s/as or NSH3 s/as (Supplementary Table 1). In addition, they carry T-DNA insertions in both genes which can be detected by combinations of the SAIL T-DNA specific primer LB3 and NSH3 specific primer NSH3as and of the SALK T-DNA specific primer LB335 and ENT3 specific primer ENT3s. To check for the absence of ENT3 and NSH3 transcripts in the corresponding double mutants, cDNA was prepared and expression of ENT3 and NSH3 was quantified by quantitative RT-PCR as described below with ENT3 and NSH3 specific primer pairs (RT-ENT3, RT-NSH3). UBQ10 served as single reference gene in this experiment. All primers used are listed (Supplementary Table 1).

\section{Biochemical Analyses}

Isolation of apoplastic sap for enzymatic and metabolite analyses was performed using 6 week old plants as described by Ziegler et al. (2000). Briefly, leaves including the petiole were vacuum infiltrated with $250 \mathrm{mM} \mathrm{KCl}$ and subsequently dried using paper. 
About 20 leaves were arranged in a $5 \mathrm{ml}$ pipette tip with the cut ends facing down and centrifuged in a $15 \mathrm{ml}$ Greiner tube at $350 \mathrm{~g}$ for $15 \mathrm{~min}$. Immediately after isolation, apoplastic sap was incubated with $\left[{ }^{14} \mathrm{C}\right]$-radiolabeled inosine for $0 / 60 \mathrm{~min}$ at $30^{\circ} \mathrm{C}$. The reaction was terminated by heating the protein to $98^{\circ} \mathrm{C}$ for $5 \mathrm{~min}$. Thin-layer chromatography on PEI-Cellulose F plates (Merck, Darmstadt, Germany) was used to quantify the amounts of hypoxanthine produced. Separation was performed for $60 \mathrm{~min}$ with water, and respective products were detected using a Cyclone Storage phosphorscreen (Perkin-Elmer Life Sciences, Waltham, USA). Spots were quantified using the OptiQuant Software Version 5.0 (Perkin-Elmer Life Sciences, Waltham, USA).

\section{Transcriptional Analyses}

For quantitative RT-PCR analyses leaf material of soil or hydroponically grown plants was collected. Depending on the analysis 3 week (photosynthesis) or 6 week (pathogenicity) old plants were used. Directly after harvest, leaf material was ground in liquid nitrogen and $100 \mathrm{mg}$ were used for RNA isolation (RNeasy plant mini kit, Qiagen, Hilden, Germany). To remove any contaminating DNA, samples were treated with DNase (RNase free DNase kit, Qiagen, Hilden, Germany). RNA was quantified and purity was checked photometrically. Total RNA was reverse transcribed with cDNA synthesis kit (qscript cDNA synthesis kit, Quanta Biosciences, Gaithersburg, USA). Quantitative RT-PCR was performed with the IQ SybrGreen supermix (BioRad, Munich, Germany) on a MyIQ PCR Cycler (BioRad, Munich, Germany) using the Primers listed in Supplementary Table 1. Melting curves were checked for all samples. Three biological replicates were measured and for normalization the reference genes GAPDH, 18SRNA, EF1A, UBQ10, and ACTIN (Supplementary Table 1) were included. To qualify for a reference gene in our experiments and on the basis of Remans et al. (2014) the delta $C_{t}$ across all experiments must be below 2. This criterion was fulfilled by UBQ10 and ACTIN for pathogenesis related gene analyses and GAPDH, 18SRNA, and ACTIN for analyses of photosynthesis related genes. Thus, the mean expression value was calculated for each of the genes and the geometric mean was used to normalize gene expression.

\section{Pathogenicity Assay}

B. cinerea BMM was kindly provided by Prof. Matthias Hahn, University of Kaiserslautern, Germany and grown on HA Medium (10 $\mathrm{g} \mathrm{l}^{-1}$ malt extract, $4 \mathrm{~g} \mathrm{l}^{-1}$ glucose, $4 \mathrm{~g} \mathrm{l}^{-1}$ yeast extract, $15 \mathrm{~g} \mathrm{l}^{-1}$ agar, $\mathrm{pH} 5.5$ ) at $20^{\circ} \mathrm{C}$ for 10 days. Conidiospores were harvested, filtered through glass wool and washed three times with water. Leaves of 6 week old soil or hydroponic culture grown plants were cut off, placed into a humidity saturated chamber and inoculated with suspension containing $1.25 \times 10^{5}$ conidia/ml in Gamborg medium (3 g $1^{-1}$ Gamborg B5 basal (Biochemie BV, Haarlem, Netherlands), $10 \mathrm{mM} \mathrm{KH} \mathrm{KH}_{4}, 10 \mathrm{mM}$ Glucose) for pathogenicity and expression analysis. Lesion formation was quantified after $24 \mathrm{~h}$ using ImageJ (Schneider et al., 2012). For expression analysis using quantitative RT-PCR pathogenesis related genes were studied. Besides NADPH Oxidase F (ATRBOHF, Atlg64060) which functions in ROS mediated pathogen response (Chaouch et al., 2012), the Pathogenesis related 1 gene (PR1, At2g14610) which is part of the salicylic acid depending pathogen response (Dong, 1998) and WRKY33 (At2g38470), a transcription factor inducing SA and jasmonate dependent response and a negative regulator of ABA signaling were included (Birkenbihl et al., 2012; Liu et al., 2015). Furthermore, plant defensin 1.2 (PDF1.2, At5g44420), a marker for jasmonic acid/ethylene signaling pathway (Ndamukong et al., 2007) encoding an antimicrobial peptide and Jasmonic acid-carboxyl-Methyltransferase (JMT, Atlg19640) as a key enzyme of jasmonic acid regulated plant response induced by wounding (Seo et al., 2001) and ORA59, which is regulated by WRKY33 and can induce expression of defense related genes like PDF1.2 (Pré et al., 2008) were examined.

\section{Quantification of Chlorophyll}

For determination of chlorophyll contents, $100 \mathrm{mg}$ frozen tissue was boiled at $95^{\circ} \mathrm{C}$ for $10 \mathrm{~min}$ in $1 \mathrm{ml} 80 \% \mathrm{EtOH}$. After centrifugation $(11,000 \mathrm{~g}, 5 \mathrm{~min})$ the step was repeated and supernatants were pooled. Chlorophyll contents were determined as absorbance at $652 \mathrm{~nm}$ and values corrected according to Arnon (1949).

\section{Metabolite Analyses}

For analysis of apoplastic nucleoside composition, apoplastic sap was isolated as described above and immediately used for HPLC quantification in a DIONEX system (P680 HPLC-Pump, ASI-100 Automated Sample Injector, Dionex UVD170U Detektor), UCI50 Universal Chromatography Interface (Dionex, Sunnyvale, USA) and Nucleodur 100-5 C18 ec-Column (Macherey-Nagel, Düren, Germany). As the eluent, a solution of $10 \mathrm{mM} \mathrm{K}_{2} \mathrm{PO}_{4}$, $\mathrm{pH} 5.4$ and $5.7 \mathrm{mM}$ tetrabutylammonium hydrogen sulfate with gradually increasing (0-80\%) acetonitrile, was used. For quantification of sugars, carbonic acids, anions and cations ion chromatography was performed as described by Hassler et al. (2012).

\section{Pulse-Amplitude-Modulation (PAM) Fluorometry Measurements}

A MINI-IMAGING-PAM fluorometer (Walz Instruments, Effeltrich, Germany) was used for in vivo chlorophyll A fluorescence assays on intact, 3 week old dark-adapted plants. For phenotype quantification, a light induction curve using standard settings was recorded (Schreiber et al., 2007).

\section{Transport Experiments}

To test the ability of ENT3 to transport inosine, the open reading frame (ORF) of ENT3 was cloned into the yeast expression vector pDR196 (Rentsch et al., 1995). Appropriate restriction sites EcoRI were introduced with the designed primers W303-ENT3s and W303-ENT3as by amplification of ENT3 from cDNA (Supplementary Table 1). To check for correct cloning the construct was sequenced. Subsequently the construct was transformed into yeast strain W303 (W303; Mat $\alpha$; ura3-1;his3-11; leu2-3_112; trp12; ade2-1; can1-100; YBL042c11,1902::kanMX4) obtained from EUROSCARF 
(Frankfurt, Germany). Yeast cells transformed with the empty vector pDR196 were used as control in uptake studies. Transport experiments were conducted essentially as described in Wormit et al. (2004) with $10 \mu \mathrm{M}$ final concentration of $\left[{ }^{14} \mathrm{C}\right]$-radiolabeled nucleosides.

Arabidopsis gene identifier (AGI) numbers for genes described in this article are: ENT3: At4g05120; NSH3: At5g18860.

\section{Statistical Analysis}

Statistical analyses were performed with One-way ANOVA with Bonferroni post-test GraphPad Prism version 5.00 for Windows, GraphPad Software, San Diego California USA, www. graphpad.com.

\section{RESULTS}

Homozygous T-DNA insertion mutants for equilibrative nucleoside transporter ENT3 (Traub et al., 2007) were crossed with homozygous T-DNA insertion mutants of the extracellular nucleoside hydrolase NSH3 (Jung et al., 2011). The resulting heterozygous plants were selfted, a homozygous double mutant isolated and analyzed for the disruption of both genes in a PCR screening based on genomic DNA (Figure 1A). Two ENT3NSH3 double knockout lines (ent3:nsh3, \#1, \#2) were used for all following experiments. Expression analyses via quantitative RT-PCR confirmed the absence of ENT3 and NSH3 transcripts in these mutants (Figure 1B). To support these results, validated biochemical screenings for both mutants were performed (Jung et al., 2011) NSH3 was identified as the sole apoplastic enzyme with activity to convert inosine to the corresponding nucleobase hypoxanthine (Jung et al., 2011). To corroborate the lack of this enzyme activity in ent3:nsh3 mutants, isolated apoplastic sap was incubated with radiolabeled inosine for $60 \mathrm{~min}$ and screened for a conversion to hypoxanthine by thin layer chromatography. Only in extracts from control plants an accumulation of hypoxanthine was determined after 60 min (Figure 1C), clearly indicating the loss of inosine hydrolase activity in nsh3-1 (Jung et al., 2011) and both ent3:nsh3 lines. To show blockage of apoplastic catabolism
A

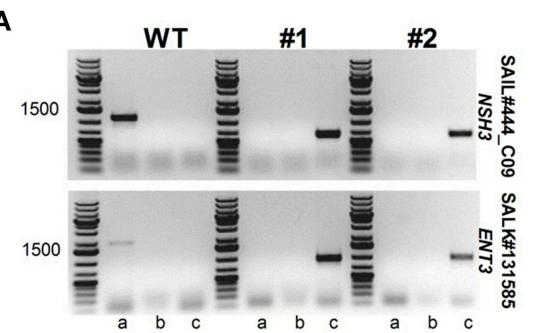

C
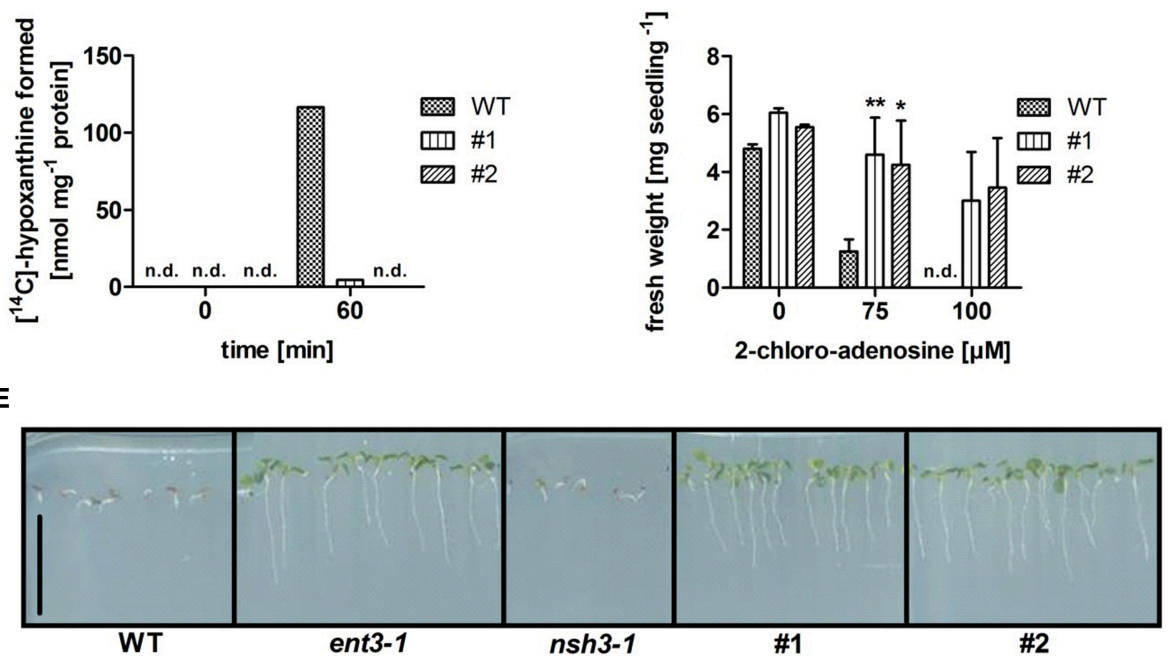

FIGURE 1 | Characterization of homozygous Arabidopsis thaliana equilibrative nucleoside transporter 3 (ENT3; SALK_131585) and nucleoside hydrolase 3 (NSH3; SAIL444_C09) double knockout mutants (\#1; \#2). (A) PCR analysis on gDNA of wild-type (WT) and homozygous double knockout mutants using gene- (a) and T-DNA-specific primers (b; c) for both possible directions of the T-DNA insertion. (B) Analysis of NSH3 and ENT3 expression in in WT and ent3:nsh3 mutants. UBQ10 expression was used as a reference. Data represent means \pm SE of three biological replicates. (C) Inosine hydrolysis assay using leaf apoplastic sap incubated with $\left[{ }^{14} \mathrm{C}\right]$-radiolabeled inosine. For zero time point determination samples were heat inactivated immediately after mixing. Experiments were repeated twice with similar results. (D) Fresh weight analysis of WT and mutants seedlings grown agar medium containing 2-chloro-adenosine $(0,75,100 \mu \mathrm{M})$ for 7 days. Data represent means $\pm S E$ of at least 16 biological replicates. Differences between lines were analyzed with One-way ANOVA and a Bonferroni correction at the 95\% confidence level. ${ }^{*} P<0.05,{ }^{* *} P<0.005$. (E) Growth of WT, ent3-1, nsh3-1, and ent3:nsh3 (\#1, \#2) plants grown on agar medium containing $200 \mu \mathrm{M}$ 5-fluoro-uridine for 14 days. Scale bar $1 \mathrm{~cm}$. n.d., not detectable. 
and nucleoside uptake in ent3:nsh3 plants, resistance toward the cytotoxic adenosine analog 2-chloro-adenosine (CADO) was analyzed. Indeed, after 10 days of growth in the presence of $75 \mu \mathrm{M}$ CADO, ent3:nsh3 plants showed almost no reduction in fresh weight (FW), whereas control plants exhibited a marked $74 \%$ fresh weight loss (Figure 1D). In a similar screen with 5fluoro-uridine, the same degree of resistance for both ent3:nsh3 lines was observed as already before for ent3-1 (Figure 1E; Traub et al., 2007).

Growth of ent3:nsh3 mutants on soil or standard MS agar plates revealed no differences in development compared to controls. This observation is supported by unchanged chlorophyll levels of seedlings grown in liquid culture under standard conditions (Figure 2A). However, when inosine, which serves as a substrate for both ENT3 (Supplementary Figure 1) and NSH3 (Jung et al., 2011) was present as sole nitrogen source, chlorophyll levels of both mutants were reduced more than 75\% (\#1: $0.00596 \mathrm{mg} \mathrm{gFW}^{-1}$; \#2: $0.00602 \mathrm{mg} \mathrm{gFW}^{-1}$ ) compared to controls (WT: $0.0254 \mathrm{mg} \mathrm{gFW}^{-1}$; Figure 2A). These results were also reflected in the fresh weights of the analyzed mutants where inosine as sole nitrogen source promoted growth of control plants to $5.3 \mathrm{mg}$ seedling $^{-1}$ whereas ent3:nsh3 \#1 exhibited $3.6 \mathrm{mg}$ seedling ${ }^{-1}$ and ent3:nsh3 \#2 $3.7 \mathrm{mg}$ seedling $^{-1}$ (Figure 2B). In contrast, adenosine or uridine provided as sole nitrogen source did not promote seedling growth in any of the lines.

To obtain a more complete picture about physiological processes in the mutants, 4 week old soil and hydroponically grown plants were monitored for soluble sugar, anion, cation and carboxylic acid contents in leaves. Even if metabolite contents differed with regard to growth conditions, no changes were detectable within the single plant lines (Supplementary Figure 2). Only in case of citric acid significant differences were observed. In both soil grown ent3:nsh3 lines citrate levels were increased 30 and $42 \%$ above control levels (Supplementary Figure 2). This effect could not be detected for plants grown in hydroponic culture.

It could be shown that ent3:nsh 3 plants are impaired in uptake and catabolism of supplied nucleosides. Therefore, one can assume an accumulation of these substances in the apoplastic space. To test this, apoplastic sap was collected from mature leaves and analyzed by HPLC. Clearly, uridine (uri, \#1: 2.56fold; \#2: 3.13-fold) and adenosine levels (ado, \#1: 3.49-fold; \#2: 4.48-fold) increased in apoplastic sap from ent3:nsh3 plants compared to controls (Figure 3). In contrast to soil grown plants, hydroponically grown plants cultivated in media lacking any organic compounds did not show accumulation of nucleosides in apoplastic fluid above control levels (Figure 3).

NSH3 expression was shown to increase after wounding or jasmonic acid (JA) treatment (Jung et al., 2011). Like wounding, also the response to necrotrophic pathogens like Sclerotinia sclerotiorum (Wang et al., 2015) and B. cinerea (Aubert et al., 2015) can induce JA signaling pathways. As a consequence, pathogenicity tests were performed with the necrotrophic fungus $B$. cinerea BMM, as this was shown to be well-suited for Arabidopsis infection (Zimmerli et al., 2001). For this, droplets of conidiospore suspensions were spotted onto leaves cut from 6 week old healthy plants. Subsequently, the leaves were incubated in a humid chamber and lesion formation was quantified $24 \mathrm{~h}$ after application of spores. Double mutant ent3:nsh3 lines exhibited a 1.5-fold increase in mean lesion size in comparison control leaves (Figure 4). When the same experiment was performed with hydroponically grown plants, no alterations in lesion size formation were observed between the lines after $24 \mathrm{~h}$ (WT: $8.47 \pm 0.37 \mathrm{~mm}^{2}$; \#1: $8.23 \pm 0.64 \mathrm{~mm}^{2}$; \#2: $7.84 \pm 0.28 \mathrm{~mm}^{2}$ ).

As necrotrophic pathogens rely on killing host cells to receive nutrients for growth, plants developed several defense strategies. Thus, resistance of Arabidopsis against necrotrophs like $B$. cinerea appears to be under complex genetic control (Rowe and Kliebenstein, 2008). This results in tremendous reprogramming of the host transcriptome (AbuQamar et al., 2006), including key regulators like transcription factors. Thus, transcripts of defense related genes were quantified in leaves challenged with $B$. cinerea using quantitative RT-PCR. Control leaves were analyzed $0 \mathrm{~h}$ after infection and after $24 \mathrm{~h}$ treated with buffer alone (Mock; Figure 5). The detection of Botrytis ACTIN transcripts confirmed the successful infection of all analyzed leaves $24 \mathrm{hpi}$, but was absent in Mock treated samples (data not shown).

All examined genes except for ATRBOHF and JMT showed increased expression levels in leaves of soil and hydroponically
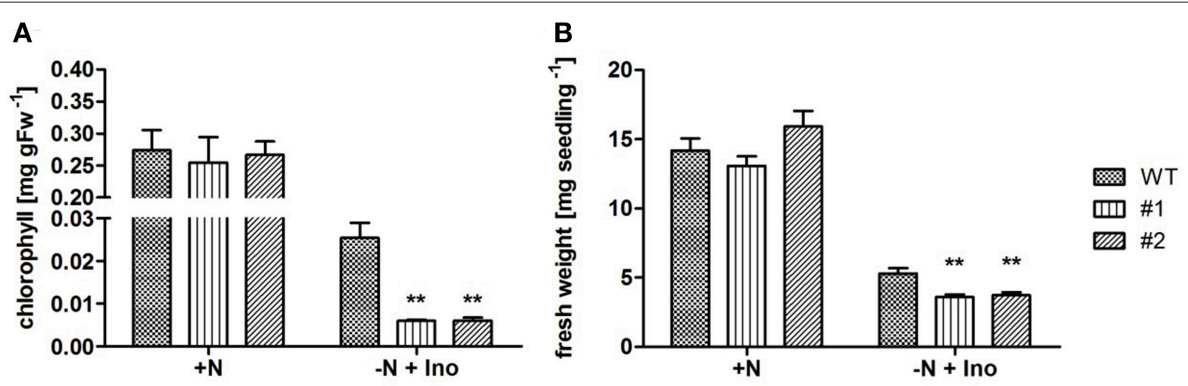

FIGURE 2 | Effects of inosine feeding on chlorophyll contents and seedling fresh weight production under conditions of limited nitrogen accessibility. Analysis of chlorophyll contents (A) and fresh weight (B) of 21 (A) and 14 (B) day old WT and ent3:nsh3 plants (\#1, \#2) grown in standard liquid culture with nitrogen $(+\mathrm{N})$ or nitrogen deprivation but supplemented with $1 \mathrm{mM}$ inosine $(-\mathrm{N}+\mathrm{Ino})$. Data represent means $\pm S E$ of four biological replicates. Differences between lines were analyzed with One-way ANOVA and a Bonferroni correction at the $95 \%$ confidence level. ${ }^{* \star} P<0.005$. 


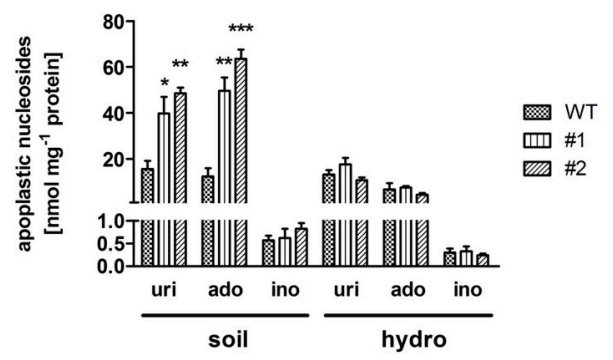

FIGURE 3 | Uridine, adenosine, and inosine contents in isolated apoplastic extracts. Plants were grown under ambient conditions on soil (soil) or hydroponic culture (hydro) for 6 weeks before nucleoside composition of the apoplastic extract was determined by high performance liquid chromatography. uri, uridine; ado, adenosine; ino, inosine. Data represent means $\pm S E$ of three biological replicates. Differences between lines were analyzed with One-way ANOVA and a Bonferroni correction at the 95\% confidence level. ${ }^{\star} P<0.05 ;{ }^{\star \star} P<0.005,{ }^{* * *} P<0.001$

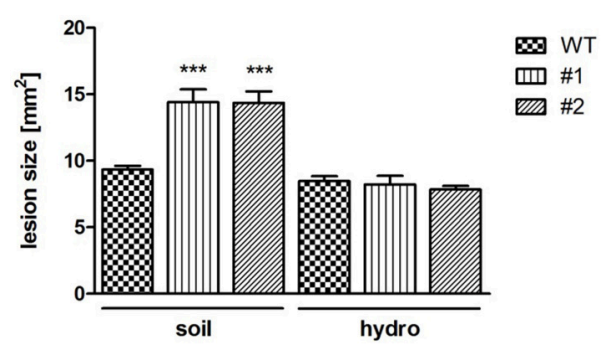

FIGURE 4 | Botrytis cinerea BMM infection caused lesion formation. Lesion formation after inoculation with $B$. cinerea BMM conidiospores at ent3:nsh3 double knockout plants grown on soil (soil) and hydroponic culture (hydro) was monitored after $24 \mathrm{~h}$. Data represent means \pm SE of at least 64 (soil) and 31 (hydro) biological replicates. Differences between lines were analyzed with One-way ANOVA and a Bonferroni correction at the 95\% confidence level. ${ }^{* \star \star} P<0.001$.

grown plants after pathogen treatment indicating the activated plant response to pathogen challenge (Figure 5B). In line with this, no significant change in transcript levels of all analyzed genes could be detected between $0 \mathrm{~h}$ control and $24 \mathrm{~h}$ Mock conditions. Transcript levels for regulators of the JA coordination and ethylene (ET) defense (ORA59, McGrath et al., 2005), redox homeostasis (ATRBOHF, NADPH oxidase, Chaouch et al., 2012), JA response (JMT, JA-Carboxyl-Methyltransferase, Seo et al., 2001; PDF1.2, plant defensin 1.2, Meng et al., 2013) and phytoalexin synthesis (PAD3, Ferrari et al., 2007) did not show significant differences between WT and mutant lines. This finding is independent of the plant growth conditions. The most marked differences in expression between wild-type and mutant lines were observed for WRKY33 and PR1 (pathogenicity related protein 1, Dong, 1998; Spoel et al., 2003) 24 hpi in leaves of soil grown plants (Figure 5B). In both cases the mutants showed significantly reduced expression. Although in wildtype and mutant plant leaves an increased WRKY33 expression was detected compared to control conditions (WT: 9.59-fold), the increase was less pronounced in both mutant lines (\#1:

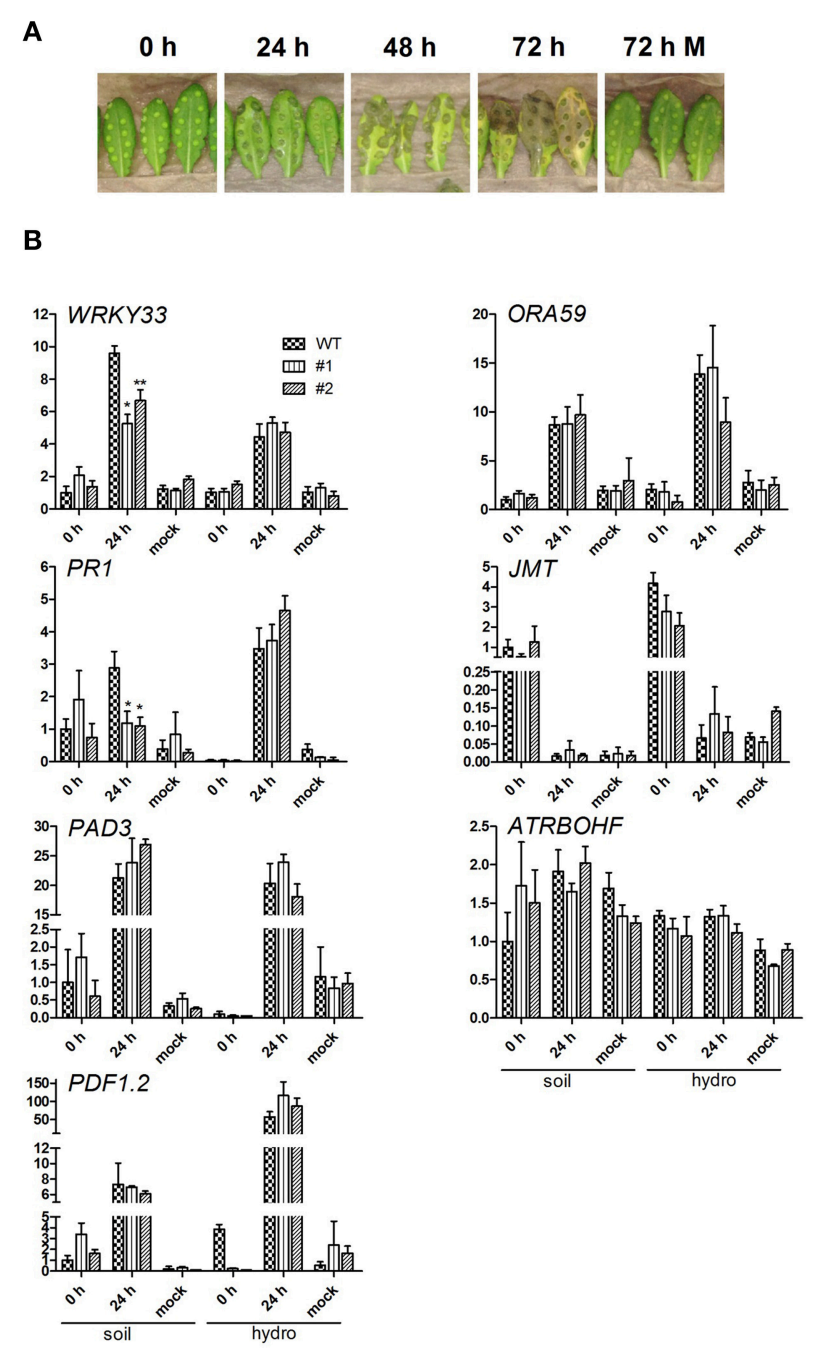

FIGURE 5 | Botrytis cinerea infection and expressional analysis of pathogenesis related transcripts. (A) Representative infection study using B. cinerea BMM condiospores infecting 6 week old, soil grown wild-type plants. The infection studies were performed using WT and both ent3:nsh3 double knockout lines grown on soil and hydroponic culture. Conidiospore and mock inoculated leaves were documented 0 and 24 hpi before used for RNA extraction. (B) For quantitative RT-PCR analysis of WRKY33, PR1, PAD3, PDF1.2, ORA59, JMT, and ATRBOHF in the context of B. cinerea BMM infection, five biological replicates per condition were used. The expression was normalized using the geometric meridian of the housekeeping genes EF1 $\alpha$ and $U B Q 10$ and represents the relative expressional change compared to respective expression of WT plants grown on soil $\mathrm{O} h$ after infection. Differences between lines were analyzed with One-way ANOVA and a Bonferroni correction at the $95 \%$ confidence level. ${ }^{\star} P<0.05 ;{ }^{* \star} P<0.005$.

2.53-fold; \#2: 4.86-fold). PR1 expression was not significantly altered 24 hpi in mutants compared to both control conditions, while the transcript increased in wild-type plant leaves 2.9-fold above the $0 \mathrm{~h}$ level (Figure 5B). No expressional alterations were detected in hydroponically grown lines (Figure 5B). WRKY33 was identified as a superior regulator during challenge with $B$. cinerea, involved in modulation of JA- and ET-mediated defense mechanisms, salicylic acid (SA) signaling and of redox 
homeostasis genes (Zheng et al., 2006; Birkenbihl et al., 2012). The data clearly indicates, that at preinfectious stages the transcripts of the examined pathogenesis related genes in soil as well as hydroponically grown plants do not differ per se. Thus, the significantly changed expression of WRKY33 and PR1 depends on the growth condition.

To elucidate further effects of the extracellular accumulation of nucleosides in ent3:nsh3 mutants, photosynthetic processes, particularly the photosynthetic performance as photosystem II efficiency $\left(\triangle \mathrm{FM} / \mathrm{Fm}^{\prime}\right)$ was analyzed (Figures $\left.6 \mathbf{A}, \mathbf{B}\right)$. A reduced PSII efficiency could be observed for ent3-1 (-9.1\%). In ent3:nsh3 mutants this value was even more decreased by $15 \%(\# 1)$ and $18 \%$ (\#2) when grown on soil (Figure 6B). In contrast, mutant plants grown in hydroponic culture exhibited no alterations of PSII efficiency in comparison to control plants (Figures 6A,B). When monitoring the expression pattern of twelve nuclear or plastid encoded photosynthesis related genes, it turned out that transcripts of PSAC (\#1: 2.1-fold; \#2: 1.91-fold), PSBA (\#1: 1.85fold; \#2: 2.12-fold) and RBCL (\#1: 1.89-fold; \#2: 2.09-fold) were specifically upregulated in soil grown mutants, but not in wildtypes or hydroponically grown mutants (Figure 6C). For PSAD a significant decreased transcript level could be detected for both soil grown mutants (\#1: 0.63-fold; \#2: 0.80-fold, Figure 6C). All other genes analyzed did not exhibit significant expression differences (Supplementary Figure 3).

\section{DISCUSSION}

\section{Double Knockouts of ENT3 and NSH3 Show Accumulation of Extracellular Nucleosides}

Research over the last years identified nucleosides as valuable metabolic intermediates involved in different aspects of plant metabolism (Jung et al., 2009, 2011; Cornelius et al., 2012; Girke et al., 2014). Here, we describe the generation of a mutant lacking the main nucleoside import activity as well as extracellular nucleoside hydrolase activity. This ent3:nsh3 double mutant combines characteristics of corresponding single mutants as increased resistance against the toxic purine and pyrimidine nucleoside analogs 2-chloro-adenosine and 5-fluoro-uridine, a diminished capacity to degrade extracellular purine nucleosides and a reduced ability to grow on inosine as sole nitrogen source. Inosine is a preferred substrate for ENT3 and NSH3. For white spruce embryos it was shown that inosine, in contrast to adenosine, is catabolized almost completely (Ashihara et al., 2001). These results are in line with a lower activity of NSH3 and cytosolic NSH1 for adenosine compared to inosine (Jung et al., 2009, 2011). As inosine cannot be converted to xanthosine directly (Dahncke and Witte, 2013), it first has to become deribosylated to hypoxanthine either in the apoplast by NSH3 or after import via ENT3 by NSH1 to become further catabolized to xanthine by xanthine dehydrogenase (Hesberg et al., 2004). Apoplastic hypoxanthine can subsequently be imported by plasma membrane located NAT3 and NAT12 transport proteins (Niopek-Witz et al., 2014). When direct import and extracellular catabolism are not active in ent3:nsh 3 mutants, inosine cannot
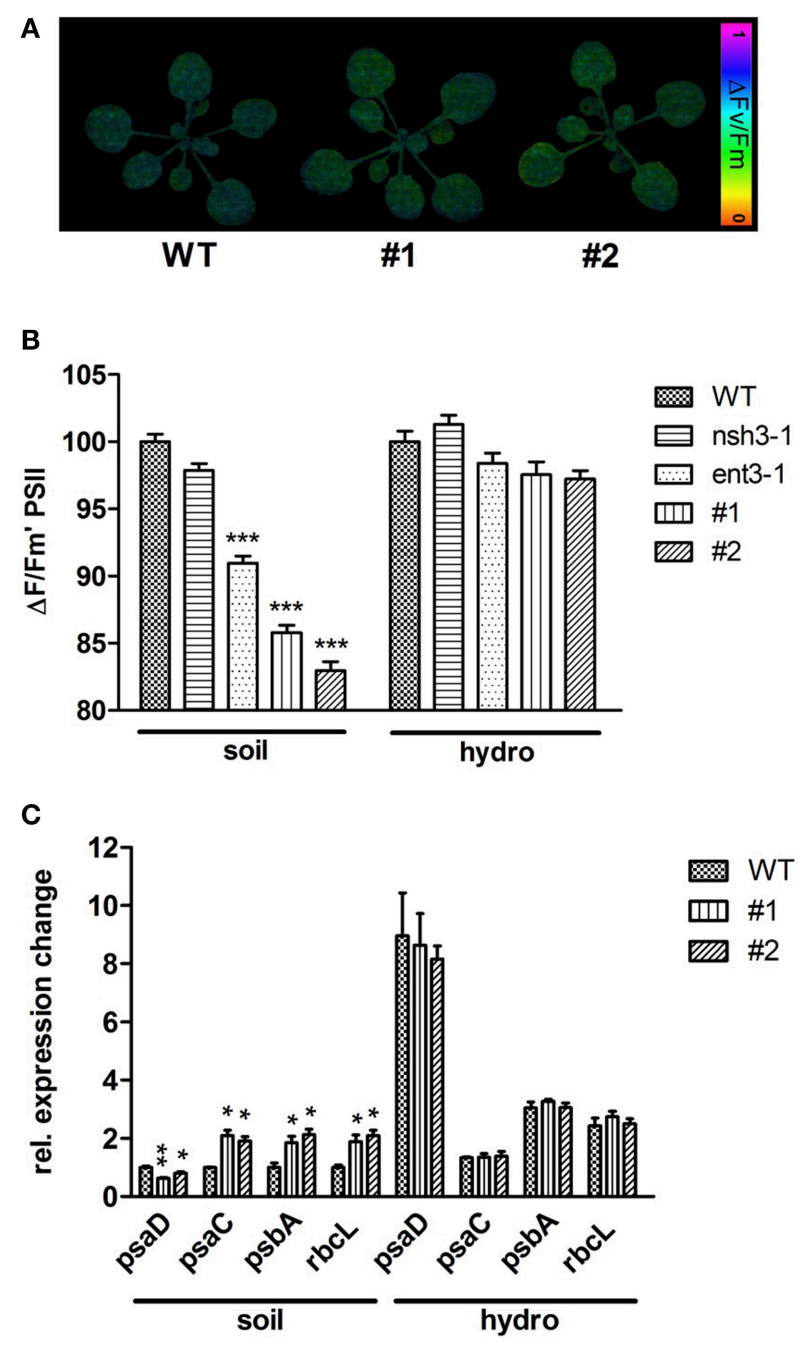

FIGURE 6 | Photosynthetic efficiency and expressional analysis of photosynthesis related genes. (A) PSIl efficiency of 3 week old WT, ENT3, and $N S H 3$ single and double knockout mutants grown on soil and hydroponic culture was examined using a MINI-IMAGING-PAM fluorometer (Walz Instruments, Effeltrich, Germany). Altered PSII efficiencies ( $\triangle \mathrm{FM} / \mathrm{Fm}$ ') were indicated by color changes. (B) Quantification of PSIl efficiency. Data represent means $\pm S E$ of at least 10 plants. Differences between lines were analyzed with One-way ANOVA and a Bonferroni correction at the 95\% confidence level $\left.{ }^{\star \star \star} P<0.001\right)$. (C) Expressional analysis of photosynthesis related genes using quantitative RT-PCR. The expression of PSAC, PSAD, PSBA and RBCL was normalized using the geometric meridian of the housekeeping genes $G A P D H$, $A C T I N$, and 18SRNA. Data represent means \pm SE of three biological replicates. Differences between lines were analyzed with One-way ANOVA and a Bonferroni correction at the $95 \%$ confidence level. ${ }^{*} P<0.05$; ${ }^{* \star} P<0.005$.

function as efficient nitrogen source. Whereas uridine and adenosine accumulated in apoplastic leaf extracts of soil grown mutant plants two- to five-fold above the level of control plants, inosine levels were not altered. Therefore, one can assume that at least quantitatively inosine plays a minor role in nucleotide metabolism in Arabidopsis leaves.

Ent3:nsh3 mutants were used as a tool to dissect the effect of reduced nucleoside import accompanied by apoplastic 
nucleoside accumulation. Nucleosides can either be taken up from the soil (Traub et al., 2007; Cornelius et al., 2012) where they can appear in respectably high amounts (Phillips et al., 1997) or originate from liberation within the plant. When comparing apoplastic nucleoside levels from soil grown plants with plants of the same size grown in fully anorganic hydroponic solution, two observations could be made: First, control plants show a similar, basal accumulation of nucleosides under both conditions. As the apoplastic fluid was free of contaminating cytosolic marker enzyme (glyceraldehyde-3-phosphate dehydrogenase) activity (data not shown), these nucleosides are not regarded as contaminations but as originating from endogenous sources. Second, the marked increase of apoplastic nucleosides in soil grown ent3:nsh 3 mutants was not observed in hydroponically grown plants. This supports the view that at least parts of the extracellular nucleosides might originate from the rhizosphere when plants are grown on soil. We aimed to test this hypothesis by feeding nucleosides to hydroponic culture and observed fast bacterial growth in the (otherwise anorganic) medium. Sterile growth on agar plates represents an alternative strategy. However, sterile culture is so much different from the applied soil and hydroponic culture and furthermore infection assays and photosynthesis measurements are hardly possible. Therefore, we decided not to perform sterile culture experiments and postpone the clarification of the origin of apoplastic nucleosides to future studies.

\section{Physiological Consequences of Extracellular Accumulation of Nucleosides-Photosynthetic Efficiency}

As a general measure of plant fitness the photosynthetic efficiency of ent3:nsh 3 mutants and controls was determined as $\triangle \mathrm{FM} / \mathrm{Fm}^{\prime}$. Clearly, PSII efficiency was significantly higher under conditions where nucleosides could be imported i.e., in control plants. Such positive effects of supplied nucleosides were also observed for Ricinus communis cotyledones where adenosine supplementation supported seedling growth (Flörchinger et al., 2006). Restricted NSH3 activity alone does not lead to reduced PSII efficiency, only when ENT3 is missing at the same time this effect can be observed. Therefore, co-limiting adenosine cleavage in addition to omitted import seems to be causative for the observed reduction in PSII efficiency. As uridine is no substrate for NSH3, adenosine is probably the most effective metabolite in these experiments.

The reduced PSII efficiency is not due to a general downregulation of photosynthesis related transcripts as known for the biotic stress response (Bilgin et al., 2010; Windram et al., 2012). In contrast, PSAC, RBCL, and PSBA transcripts, all plastid encoded, were significantly more abundant in soil grown mutants whereas nuclear encoded PSAD transcripts were lowered. It is therefore unlikely that reduced PSII efficiency is caused by transcriptional downregulation of corresponding genes. It is more likely that other, so far unknown effectors act on photosynthesis and the observed plants response might be a compensation reaction. In the typical response to pathogen challenge downregulation of photosynthetic gene expression is assumed to enable the plant to reallocate nitrogen resources for synthesis of new defense proteins (Windram et al., 2012). As ent3:nsh 3 mutants do not show altered transcript levels of photosynthesis related genes per se, a regulation different from the pathogen response pathways is more likely.

Reduced PSII efficiency was not reflected in changes in sugar, anion, or cation contents on a whole leaf basis. However, among the carboxylic acids, citrate levels increased slightly but significantly in double mutant plants. A generally repressive effect of citrate on the abundance of photosynthetic transcripts was shown (Finkemeier et al., 2013). However, whether this links increased citrate levels to reduced PSII efficiency in our mutants remains elusive so far.

\section{Physiological Consequences of Extracellular Accumulation of Nucleosides-Response to $B$. cinerea Challenge}

Extracellular metabolites such as extracellular ATP (eATP) and NAD have been identified as danger signals or elicitors and play a role in the plant response to pathogen attack (Zhang and Mou, 2009; Cao et al., 2014; Tanaka et al., 2014). In addition, extracellular adenosine was shown to attenuate ATP induced generation of reactive oxygen species (Song et al., 2006). Therefore, we analyzed the response of ent3:nsh 3 mutants toward infection with the necrotrophic, highly virulent $B$. cinerea BMM strain. Lesion formation significantly increased in both ent3:nsh3 mutants when grown on soil. However, when plants were grown hydroponically where no nucleosides accumulate in the apoplastic space and no difference in PSII efficiency was observed, also lesion formation was indistinguishable between mutants and control plants. This is indicative of a function of extracellular nucleosides in plant response toward $B$. cinerea infection. In line with this, expression of $\mathrm{NSH}_{3}$ was shown to increase after wounding or treatment with JA, a known signaling compound in necrotrophic pathogen attack. The expression of genes involved in different plant pathogen response pathways showed no differences between mutant and control lines at time $0 \mathrm{~h}$ or Mock treatment, indicating that extracellular nucleosides themselves are not perceived as danger signals or DAMPs (damage associated molecular patterns). Exogenously administered ATP similarly does not induce PR1 expression, whereas NAD(P) does (Zhang and Mou, 2009). Obviously, nucleosides and purine nucleotides exert different effects as pyridine nucleotides.

The expression of ATRBOHF, PAD3, ORA59, and PDF1.2 increased upon pathogen treatment in soil and hydroponically grown plants, but remained indistinguishable between mutants and controls. However, the increase in PR1 and WRKY33 transcripts in ent3:nsh3 soil grown plants accounted only to $50 \%$ of the transcript increase measured in wild-type plants. Interestingly, reduced WRKY33 expression in knockout lines causes decreased pathogen resistance and an elevated numbers of dying cells after $B$. cinerea inoculation for $40 \mathrm{~h}$ (Birkenbihl et al., 2012). Furthermore, conidiophores appeared already 3 dpi 
only on WRKY33 knockout plants but not on WT plants. This indicates the outstanding regulatory function of WRKY33 on the plant response during pathogen challenge. It is easy to assume, that altered expression of this key player in ent3:nsh 3 knockout plants could also be causative for increased pathogen expansion, indicated by enlarged lesion formation. It is further known that WRKY33 binds to PAD3 promotor regions after being released from a trimeric complex (Andreasson et al., 2005; Qiu et al., 2008) regulating the synthesis of the phytoalexin camalexin, a critical component for plant defense mechanisms. Mutants deficient in PAD3 are highly susceptible to B. cinerea (Ferrari et al., 2007). Furthermore, ORA59, a transcription factor of the AP2-ERF family, is involved in the regulation of JA and ET defense genes (McGrath et al., 2005) and is positively regulated by WRKY33 (Birkenbihl et al., 2012). Also the expression of PDF1.2 depends on the presence of WRKY33. These examples additionally highlight the outstanding role of the WRKY transcription factor and its broad regulatory functions in pathogen response pathways. Although there is no respective response for $P A D 3$, ORA59, and PDF1.2 expression in our experiments as seen for WRKY33 knockout plants (Birkenbihl et al., 2012), it can be suggested, that the alteration of WRKY33 transcript levels has wide spreading influences on general pathogen response pathways.

Besides influences on ATRBOHF, PAD3, ORA59, and PDF1.2 expression, it was shown that WRKY33 negatively regulates synthesis of SA, a major signaling component critical for the development of systemic acquired resistance and the induction of PR genes (Malamy et al., 1990; Métraux et al., 1990). Thus, in WRKY33 knockout mutants a higher PR1 expression could be detected resulting in three times higher SA accumulation $24 \mathrm{~h}$ after infection with $B$. cinerea. This means that WRKY33 is involved in preventing the inappropriate induction of the SA pathway during the challenge with necrotrophic pathogens. This alteration of transcript can't be seen in our experiments as reduced WRKY33 expression is accompanied with decreased $P R 1$ transcript levels, pointing to a reduced SA dependent defense reaction. As WRKY33 is also involved in JA induced plant defense, JA dependent signaling might be compromised in ent3:nsh3. This observation is in line with the described increased expression of NSH3 by JA (Jung et al., 2011).

\section{REFERENCES}

AbuQamar, S., Chen, X., Dhawan, R., Bluhm, B., Salmeron, J., Lam, S., et al. (2006). Expression profiling and mutant analysis reveals complex regulatory networks involved in Arabidopsis response to Botrytis infection. Plant J. 48, 28-44. doi: 10.1111/j.1365-313X.2006.02849.x

Andreasson, E., Jenkins, T., Brodersen, P., Thorgrimsen, S., Petersen, N. H., Zhu, S., et al. (2005). The MAP kinase substrate MKS1 is a regulator of plant defense responses. EMBO J. 24, 2579-2589. doi: 10.1038/sj.emboj.76 00737

Arnon, D. I. (1949). Copper enzymes in isolated chloroplasts. Polyphenol oxidase in Beta vulgaris. Plant Physiol. 24, 1-15. doi: 10.1104/pp.24.1.1

Ashihara, H., Stasolla, C., Loukanina, N., and Thorpe, T. A. (2001). Purine metabolism during white spruce somatic embryo development: salvage of
The ent3:nsh3 mutant response at the level of gene expression (photosynthesis related genes and pathogenesis related genes) differs from the normal response toward biotic stress and points to an interference with related signaling cascades. Supporting results for this view come from the analysis of the OXT1 mutant exhibiting altered adenine phosphoribosyltransferase 1. In this mutant moderately increased cellular adenine contents (1.5fold) lead to markedly increased resistance against oxidative stress (Sukrong et al., 2012). Thus, altered (purine) nucleobase or nucleoside levels may act as physiological signals affecting stress responses in Arabidopsis. Adaptation of ENT3 and NSH3 activity to environmental conditions are obviously required to balance nucleobase and nucleoside levels to allow for optimal stress response. In animals, both ATP and adenosine are known to function as extracellular signaling molecules. A plant receptor for eATP has been identified recently with DORN1 (Choi et al., 2014) whereas receptors for adenosine, other nucleosides or nucleobases are unknown so far. However, DORN1 is a member of plant specific lectin receptor kinases which comprise a large gene family. Whether any lectin receptor kinase recognizes nucleosides or nucleobases has to be shown in future experiments.

\section{AUTHOR CONTRIBUTIONS}

Conceptualization, TM; Methodology, MD; Investigation, MD, MF, SN, CG, Writing-Original Draft, TM; Writing-Review and Editing, MD, CG.

\section{ACKNOWLEDGMENTS}

This work was supported by DFG-grant MO 1032/3-2 and IRTG 1830. We thank Prof. Matthias Hahn for supporting pathogen experiments. We gratefully acknowledge general support of the work by Prof. H. Ekkehard Neuhaus.

\section{SUPPLEMENTARY MATERIAL}

The Supplementary Material for this article can be found online at: http://journal.frontiersin.org/article/10.3389/fpls.2015. 01158

adenine, adenosine, and inosine. Plant Sci. 160, 647-657. doi: 10.1016/S01689452(00)00441-6

Aubert, Y., Widemann, E., Miesch, L., Pinot, F., and Heitz, T. (2015). CYP94mediated jasmonoyl-isoleucine hormone oxidation shapes jasmonate profiles and attenuates defence responses to Botrytis cinerea infection. J. Exp. Bot. 66, 3879-3892. doi: 10.1093/jxb/erv190

Buchanan, B. B., Gruissem, W., and Jones, R. L. (2002). Biochemistry and Molecular Biology of Plants: American Society of Plant Physiologists. Hoboken: Wiley \& Sons.

Bilgin, D. D., Zavala, J. A., Zhu, J., Clough, S. J., Ort, D. R., and DeLucia, E. H. (2010). Biotic stress globally downregulates photosynthesis genes. Plant Cell Environ. 33, 1597-1613. doi: 10.1111/j.1365-3040.2010.02167.x

Birkenbihl, R. P., Diezel, C., and Somssich, I. E. (2012). Arabidopsis WRKY33 is a key transcriptional regulator of hormonal and metabolic 
responses toward Botrytis cinerea infection. Plant Physiol. 159, 266-285. doi: 10.1104/pp.111.192641

Borderies, G., Jamet, E., Lafitte, C., Rossignol, M., Jauneau, A., Boudart, G., et al. (2003). Proteomics of loosely bound cell wall proteins of Arabidopsis thaliana cell suspension cultures: a critical analysis. Electrophoresis 24, 3421-3432. doi: 10.1002/elps.200305608

Cao, Y., Tanaka, K., Nguyen, C. T., and Stacey, G. (2014). Extracellular ATP is a central signaling molecule in plant stress responses. Curr. Opin. Plant Biol. 20, 82-87. doi: 10.1016/j.pbi.2014.04.009

Chaouch, S., Queval, G., and Noctor, G. (2012). AtRbohF is a crucial modulator of defence-associated metabolism and a key actor in the interplay between intracellular oxidative stress and pathogenesis responses in Arabidopsis. Plant J. 69, 613-627. doi: 10.1111/j.1365-313X.2011.04816.x

Choi, J., Tanaka, K., Cao, Y., Qi, Y., Qiu, J., Liang, Y., et al. (2014). Identification of a plant receptor for extracellular ATP. Science 343, 290-294. doi: $10.1126 /$ science.343.6168.290

Conn, S. J., Hocking, B., Dayod, M., Xu, B., Athman, A., Henderson, S., et al. (2013). Protocol: optimising hydroponic growth systems for nutritional and physiological analysis of Arabidopsis thaliana and other plants. Plant Methods 9:4. doi: 10.1186/1746-4811-9-4

Cornelius, S., Traub, M., Bernard, C., Salzig, C., Lang, P., and Möhlmann, T. (2012). Nucleoside transport across the plasma membrane mediated by equilibrative nucleoside transporter 3 influences metabolism of Arabidopsis seedlings. Plant Biol. 14, 696-705. doi: 10.1111/j.1438-8677.2012.00562.x

Dahncke, K., and Witte, C. P. (2013). Plant purine nucleoside catabolism employs a guanosine deaminase required for the generation of xanthosine in Arabidopsis. Plant Cell 25, 4101-4109. doi: 10.1105/tpc.113.117184

Dong, X. (1998). SA, JA, ethylene and disease resistance in plants. Curr. Opin. Plant Biol. 1, 316-323. doi: 10.1016/1369-5266(88)80053-0

Ferrari, S., Galletti, R., Denoux, C., De Lorenzo, G., Ausubel, F. M., and Dewdney, J. (2007). Resistance to Botrytis cinerea induced in Arabidopsis by elicitors is independent of salicylic acid, ethylene, or jasmonate signaling but requires phytoalexin deficient 3. Plant Physiol. 144, 367-379. doi: 10.1104/pp.107.095596

Finkemeier, I., König, A. C., Heard, W., Nunes-Nesi, A., Pham, P. A., Leister, D., et al. (2013). Transcriptomic analysis of the role of carboxylic acids in metabolite signaling in Arabidopsis leaves. Plant Physiol. 162, 239-253. doi: 10.1104/pp.113.214114

Flörchinger, M., Zimmermann, M., Traub, M., Neuhaus, H. E., and Möhlmann, T. (2006). Adenosine stimulates anabolic metabolism in developing castor bean (Ricinus communis L.) cotyledons. Planta 223, 340-348. doi: 10.1007/s00425005-0091-1

Girke, C., Daumann, M., Niopek-Witz, S., and Möhlmann, T. (2014). Nucleobase and nucleoside transport and integration into plant metabolism. Front. Plant Sci. 5:443. doi: $10.3389 /$ fpls.2014.00443

Hahn, M. (2014). The rising threat of fungicide resistance in plant pathogenic fungi: Botrytis as a case study. J. Chem. Biol. 7, 133-141. doi: 10.1007/s12154014-0113-1

Hassler, S., Lemke, L., Jung, B., Möhlmann, T., Kruger, F., Schumacher, K., et al. (2012). Lack of the Golgi phosphate transporter PHT4;6 causes strong developmental defects, constitutively activated disease resistance mechanisms and altered intracellular phosphate compartmentation in Arabidopsis. Plant J. 72, 732-744. doi: 10.1111/j.1365-313X.2012.05106.x

Hesberg, C., Hansch, R., Mendel, R. R., and Bittner, F. (2004). Tandem orientation of duplicated xanthine dehydrogenase genes from Arabidopsis thaliana: differential gene expression and enzyme activities. J. Biol. Chem. 279, 13547-13554. doi: 10.1074/jbc.M312929200

Jung, B., Flörchinger, M., Kunz, H. H., Traub, M., Wartenberg, R., Jeblick, W., et al. (2009). Uridine-ribohydrolase is a key regulator in the uridine degradation pathway of Arabidopsis. Plant Cell 21, 876-891. doi: 10.1105/tpc. 108.062612

Jung, B., Hoffmann, C., and Möhlmann, T. (2011). Arabidopsis nucleoside hydrolases involved in intracellular and extracellular degradation of purines. Plant J. 65, 703-711. doi: 10.1111/j.1365-313X.2010. 04455.x

Kwon, H. K., Yokoyama, R., and Nishitani, K. (2005). A proteomic approach to apoplastic proteins involved in cell wall regeneration in protoplasts of Arabidopsis suspension-cultured cells. Plant Cell Physiol. 46, 843-857. doi: $10.1093 / \mathrm{pcp} / \mathrm{pci0} 89$
Liu, S., Kracher, B., Ziegler, J., Birkenbihl, R. P., and Somssich, I. E. (2015). Negative regulation of ABA signaling by WRKY33 is critical for Arabidopsis immunity towards Botrytis cinerea 2100. Elife. 4:e07298. doi: 10.7554/eLife.07295

Malamy, J., Carr, J. P., Klessig, D. F., and Raskin, I. (1990). Salicylic acid: a likely endogenous signal in the resistance response of tobacco to viral infection. Science 250, 1002-1004. doi: 10.1126/science.250.4983.1002

McGrath, K. C., Dombrecht, B., Manners, J. M., Schenk, P. M., Edgar, C. I., Maclean, D. J., et al. (2005). Repressor- and activator-type ethylene response factors functioning in jasmonate signaling and disease resistance identified via a genome-wide screen of Arabidopsis transcription factor gene expression. Plant Physiol. 139, 949-959. doi: 10.1104/pp.105.068544

Meng, X., Xu, J., He, Y., Yang, K. Y., Mordorski, B., Liu, Y., et al. (2013). Phosphorylation of an ERF transcription factor by Arabidopsis MPK3/MPK6 regulates plant defense gene induction and fungal resistance. Plant Cell 25, 1126-1142. doi: 10.1105/tpc.112.109074

Métraux, J. P., Signer, H., Ryals, J., Ward, E., Wyss-Benz, M., Gaudin, J., et al. (1990). Increase in salicylic acid at the onset of systemic acquired resistance in cucumber. Science 250, 1004-1006. doi: 10.1126/science.250.4983.1004

Möhlmann, T., Steinebrunner, I., and Neuhaus, H. E. (2014). "Nucleotides and nucleosides: transport, metabolism and signaling function of extracellular ATP," in Progress in Botany, Vol. 75, eds U. Lüttge, W. Beyschlag, and J. Cushman (Springer), 119-144.

Ndamukong, I., Abdallat, A. A., Thurow, C., Fode, B., Zander, M., Weigel, R., et al. (2007). SA-inducible Arabidopsis glutaredoxin interacts with TGA factors and suppresses JA-responsive PDF1.2 transcription. Plant J. 50, 128-139. doi: 10.1111/j.1365-313X.2007.03039.x

Niopek-Witz, S., Deppe, J., Lemieux, M. J., and Möhlmann, T. (2014). Biochemical characterization and structure-function relationship of two plant NCS2 proteins, the nucleobase transporters NAT3 and NAT12 from Arabidopsis thaliana. Biochim. Biophys. Acta 1838, 3025-3035. doi: 10.1016/j.bbamem.2014.08.013

Phillips, D. A., Joseph, C. M., and Hirsch, P. R. (1997). Occurrence of flavonoids and nucleosides in agricultural soils. Appl. Environ. Microbiol. 63, 4573-4577.

Pré, M., Atallah, M., Champion, A., De Vos, M., Pieterse, C. M., and Memelink, J. (2008). The AP2/ERF domain transcription factor ORA59 integrates jasmonic acid and ethylene signals in plant defense. Plant Physiol. 147, 1347-1357. doi: 10.1104/pp.108.117523

Qiu, J. L., Fiil, B. K., Petersen, K., Nielsen, H. B., Botanga, C. J., Thorgrimsen, S., et al. (2008). Arabidopsis MAP kinase 4 regulates gene expression through transcription factor release in the nucleus. EMBO J. 27, 2214-2221. doi: 10.1038/emboj.2008.147

Remans, T., Keunen, E., Bex, G. J., Smeets, K., Vangronsveld, J., and Cuypers, A. (2014). Reliable gene expression analysis by reverse transcription-quantitative PCR: reporting and minimizing the uncertainty in data accuracy. Plant Cell 26, 3829-3837. doi: 10.1105/tpc.114.130641

Rentsch, D., Laloi, M., Rouhara, I., Schmelzer, E., Delrot, S., and Frommer, W. B. (1995). NTR1 encodes a high affinity oligopeptide transporter in Arabidopsis. FEBS Lett. 370, 264-268. doi: 10.1016/0014-5793(95)00853-2

Rowe, H. C., and Kliebenstein, D. J. (2008). Complex genetics control natural variation in Arabidopsis thaliana resistance to Botrytis cinerea. Genetics 180, 2237-2250. doi: 10.1534/genetics.108.091439

Scheible, W. R., Morcuende, R., Czechowski, T., Fritz, C., Osuna, D., PalaciosRojas, N., et al. (2004). Genome-wide reprogramming of primary and secondary metabolism, protein synthesis, cellular growth processes, and the regulatory infrastructure of Arabidopsis in response to nitrogen. Plant Physiol. 136, 2483-2499. doi: 10.1104/pp.104.047019

Schneider, C. A., Rasband, W. S., and Eliceiri, K. W. (2012). NIH Image to ImageJ: 25 years of image analysis. Nat. Methods 9, 671-675. doi: 10.1038/nmeth.2089

Schreiber, U., Quayle, P., Schmidt, S., Escher, B. I., and Mueller, J. F. (2007). Methodology and evaluation of a highly sensitive algae toxicity test based on multiwell chlorophyll fluorescence imaging. Biosens. Bioelectron. 22, 2554-2563. doi: 10.1016/j.bios.2006.10.018

Seo, H. S., Song, J. T., Cheong, J. J., Lee, Y. H., Lee, Y. W., Hwang, I., et al. (2001). Jasmonic acid carboxyl methyltransferase: a key enzyme for jasmonateregulated plant responses. Proc. Natl. Acad. Sci. U.S.A. 98, 4788-4793. doi: $10.1073 /$ pnas.081557298

Song, C. J., Steinebrunner, I., Wang, X., Stout, S. C., and Roux, S. J. (2006). Extracellular ATP induces the accumulation of superoxide via 
NADPH oxidases in Arabidopsis. Plant Physiol. 140, 1222-1232. doi: 10.1104/pp.105.073072

Spoel, S. H., Koornneef, A., Claessens, S. M., Korzelius, J. P., van Pelt, J. A., Mueller, M. J., et al. (2003). NPR1 modulates cross-talk between salicylateand jasmonate-dependent defense pathways through a novel function in the cytosol. Plant Cell 15, 760-770. doi: 10.1105/tpc.009159

Sukrong, S., Yun, K. Y., Stadler, P., Kumar, C., Facciuolo, T., Moffatt, B. A., et al. (2012). Improved growth and stress tolerance in the Arabidopsis oxt1 mutant triggered by altered adenine metabolism. Mol. Plant 5, 1310-1332. doi: $10.1093 / \mathrm{mp} / \mathrm{sss} 065$

Taki, N., Sasaki-Sekimoto, Y., Obayashi, T., Kikuta, A., Kobayashi, K., Ainai, T., et al. (2005). 12-oxo-phytodienoic acid triggers expression of a distinct set of genes and plays a role in wound-induced gene expression in Arabidopsis. Plant Physiol. 139, 1268-1283. doi: 10.1104/pp.105.067058

Tanaka, K., Choi, J., Cao, Y., and Stacey, G. (2014). Extracellular ATP acts as a damage-associated molecular pattern (DAMP) signal in plants. Front. Plant Sci. 5:446. doi: 10.3389/fpls.2014.00446

Traub, M., Flörchinger, M., Piecuch, J., Kunz, H. H., Weise-Steinmetz, A., Deitmer, J. W., et al. (2007). The fluorouridine insensitive 1 (fur1) mutant is defective in equilibrative nucleoside transporter 3 (ENT3), and thus represents an important pyrimidine nucleoside uptake system in Arabidopsis thaliana. Plant J. 49, 855-864. doi: 10.1111/j.1365-313X.2006.02998.x

Wang, C., Ding, Y., Yao, J., Zhang, Y., Sun, Y., Colee, J., et al. (2015). The Arabidopsis elongator subunit 2 positively contributes to resistance to the necrotrophic fungal pathogens Botrytis cinerea and Alternaria brassicicola. Plant J. 83, 1019-1033. doi: 10.1111/tpj.12946

Weigel, D., and Glazebrook, J. (2002). Arabidopsis. A Laboratory Manual. New York, NY: Cold Spring Harbor Laboratory Press.

Windram, O., Madhou, P., McHattie, S., Hill, C., Hickman, R., Cooke, E., et al. (2012). Arabidopsis defense against Botrytis cinerea: chronology and regulation deciphered by high-resolution temporal transcriptomic analysis. Plant Cell 24, 3530-3557. doi: 10.1105/tpc.112.102046
Wormit, A., Traub, M., Flörchinger, M., Neuhaus, H. E., and Möhlmann, T. (2004). Characterization of three novel members of the Arabidopsis thaliana equilibrative nucleoside transporter (ENT) family. Biochem. J. 383, 19-26. doi: 10.1042/BJ20040389

Zhang, X., and Mou, Z. (2009). Extracellular pyridine nucleotides induce PR gene expression and disease resistance in Arabidopsis. Plant J. 57, 302-312. doi: 10.1111/j.1365-313X.2008.03687.x

Zheng, Z., Qamar, S. A., Chen, Z., and Mengiste, T. (2006). Arabidopsis WRKY33 transcription factor is required for resistance to necrotrophic fungal pathogens. Plant J. 48, 592-605. doi: 10.1111/j.1365-313X.2006.02901.x

Ziegler, M. T., Thomas, S. R., and Danna, K. J. (2000). Accumulation of a thermostable endo-1,4- $\alpha$-D-glucanase in the apoplast of Arabidopsis thaliana leaves. Mol. Breeding 6, 37-46. doi: 10.1023/A:100966 7524690

Zimmerli, L., Métraux, J. P., and Mauch-Mani, B. (2001). beta-Aminobutyric acidinduced protection of Arabidopsis against the necrotrophic fungus Botrytis cinerea. Plant Physiol. 126, 517-523. doi: 10.1104/pp.126.2.517

Zrenner, R., Stitt, M., Sonnewald, U., and Boldt, R. (2006). Pyrimidine and purine biosynthesis and degradation in plants. Annu. Rev. Plant Biol. 57, 805-836. doi: 10.1146/annurev.arplant.57.032905.105421

Conflict of Interest Statement: The authors declare that the research was conducted in the absence of any commercial or financial relationships that could be construed as a potential conflict of interest.

Copyright (C) 2015 Daumann, Fischer, Niopek-Witz, Girke and Möhlmann. This is an open-access article distributed under the terms of the Creative Commons Attribution License (CC BY). The use, distribution or reproduction in other forums is permitted, provided the original author(s) or licensor are credited and that the original publication in this journal is cited, in accordance with accepted academic practice. No use, distribution or reproduction is permitted which does not comply with these terms. 